\title{
"What Happened Here!?" Towards a Taxonomy for User Interaction with Spatio-Temporal Game Data Visualization
}

\author{
ERICA KLEINMAN, University of California, Santa Cruz, USA \\ NIKITHA PREETHAM, Northeastern University, USA \\ ZHAOQING TENG, University of California, Santa Cruz, USA \\ ANDY BRYANT, University of California, Santa Cruz, USA \\ MAGY SEIF EL-NASR, University of California, Santa Cruz, USA
}

Currently, there is no formal taxonomy for the activities that users engage in when interacting with and making meaning from spatio-temporal game data visualizations. As data visualization, especially spatio-temporal visualization, becomes more popular for game data analytics, it becomes increasingly crucial that we develop a formal understanding of how users, especially players, interact with and extract meaning from game data using these systems. However, existing taxonomies developed for InfoVis are not directly applicable due to domain differences and a lack of consensus within the literature. This paper presents the beginnings of a taxonomy for user interaction with spatio-temporal data specific to the domain of games, developed from the results of a qualitative user study $(\mathrm{n}=7)$ in which experienced players were tasked with using a spatio-temporal visualization system to explore and understand telemetry data from Defense of the Ancients 2 (DotA 2). The taxonomy includes seven activities organized into three categories: Data Interaction, Sense Making, and Validation. We discuss the implications of these activities on design and future research.

CCS Concepts: • Human-centered computing $\rightarrow$ Empirical studies in HCI; User studies.

Additional Key Words and Phrases: Information Visualization, Data Visualization, Game Data, Telemetry Data, Spatio-Temporal Visualization, Interaction Taxonomies, Games, User Study, Think-Aloud, Qualitative Methods

\section{ACM Reference Format:}

Erica Kleinman, Nikitha Preetham, Zhaoqing Teng, Andy Bryant, and Magy Seif El-Nasr. 2021. "What Happened Here!?" Towards a Taxonomy for User Interaction with Spatio-Temporal Game Data Visualization. Proc. ACM Hum.-Comput. Interact. 5, CHI PLAY, Article 260 (September 2021), 27 pages. https://doi.org/10.1145/3474687

\section{INTRODUCTION}

Game data analytics and visualization are becoming increasingly popular within both academia and the industry due to the ease with which they allow developers and researchers to study player behavior at scale [13]. This is especially true of spatio-temporal visualizations, which permit the context sensitive analysis of granular data $[2,24]$ and afford players the opportunity to study the gameplay of others' such that they are able to reason about their decision making process,

Authors' addresses: Erica Kleinman, emkleinm@ucsc.edu, University of California, Santa Cruz, USA, 1156 High Street, Santa Cruz, California, 95064; Nikitha Preetham, preetham.n@northeastern.edu, Northeastern University, USA, 360 Huntington Ave, Boston, Massachusetts, 02115; Zhaoqing Teng, zhteng@ucsc.edu, University of California, Santa Cruz, USA, 1156 High Street, Santa Cruz, California, 95064; Andy Bryant, a@andymbryant.com, University of California, Santa Cruz, USA, 1156 High Street, Santa Cruz, California, 95064; Magy Seif El-Nasr, mseifeln@ucsc.edu, University of California, Santa Cruz, USA, 1156 High Street, Santa Cruz, California, 95064.

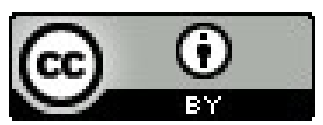

This work is licensed under a Creative Commons Attribution International 4.0 License.

(C) 2021 Copyright held by the owner/author(s).

2573-0142/2021/9-ART260. https://doi.org/10.1145/3474687

Proc. ACM Hum.-Comput. Interact., Vol. 5, No. CHI PLAY, Article 260. Publication date: September 2021. 
learn new strategies, and improve at play [1, 18, 28]. However, existing work examining spatiotemporal game data visualization has focused predominantly on how to design such systems for readability and comprehension $[63,66]$, or have only provided a high level discussion of how users interact with and make meaning from data visualization [4, 27, 34]. As a result, there is currently no formal understanding of how users, and particularly, players, interact with spatio-temporal visualizations to make sense of game data. Such an understanding would benefit the field of game data visualization by providing guidance to the designers of future systems in terms of what types of interaction they should account for and how to present the data to effectively meet players' needs.

The field of information visualization has dedicated a great deal of research to generating an understanding of how users interact with and make meaning from data, the result of which is numerous taxonomies for user interaction with information visualization [15, 55, 71]. One foundational example is Shneiderman's "Task by data type taxonomy for information visualizations" from 1996 [58], which identifies seven behavioral tasks that users engage in when exploring visualized data, including gaining an overview, examining details when needed, and relating different data points. These taxonomies have had a great influence on the field of information visualization, with Shneiderman's having over six thousand citations on Google Scholar, by informing the design of systems and providing a foundation upon which interaction schemes and their impact on users can be evaluated. Developing a taxonomy for game data visualizations would provide a similar foundation, and facilitate further study of interaction with visualized game data in the context of play.

Although insightful, the infovis work suffers from several key drawbacks that make it difficult to apply existing taxonomies directly to games, and highlight the need for game data visualization, as a domain, to develop their own taxonomy. First, existing taxonomies are predominantly based on reviews of literature or systems, rather than user studies [15, 49, 55, 70-72]. This has led to a lack of consensus within the literature that makes it difficult to select an appropriate taxonomy to adapt to game data visualization.

Second, InfoVis taxonomies rarely include the cognitive processes involved in making sense of data $[61,70,72]$ and, more importantly, they rarely account for users' need to understand the decision making processes and reasoning of those who produced the data. This is a critical omission in the context of games, where visualizations, especially player-facing tools, are often interacted with in the context of learning and mastery of play. Specifically, existing literature discusses how players learn by analyzing others' gameplay data such that they can study and adopt their decision making and strategic processes as well as compare their own play against an expert's to identify mistakes and ways to improve $[1,4,28,66,67,69]$. However, as gameplay data lacks the input of the player or commentator, who would traditionally be outlining the reasoning behind each decision on a stream or broadcast, those who use visualizations to study others' data must deduce that reasoning themselves. Such deduction is a predominantly cognitive process that involves developing a mental model of gameplay context, inferred from the visualization, that can inform an understanding of others' decision making processes. By contrast, while reflection on population data is a topic of interest [21,45], information visualization is less concerned with facilitating the study and adoption of others' decision making processes in such a granular manner. As such the cognitive processes involved in comprehending decision making are largely absent from existing taxonomies.

Third, information visualization taxonomies focus less on spatio-temporal visualizations $[15,50]$. By contrast, spatio-temporal visualizations, which are able to convey spatial and temporal context such that reasoning and decision making can be more readily deduced, have become popular for visualizing game data $[1,18,19,28,64-66]$. Existing information visualization literature that 
develops taxonomies for user interaction with information visualization does so with aggregate data and graph or chart based visualizations [30] and has further demonstrated that applying such taxonomies to spatio-temporal systems requires substantial adaptation $[15,50]$. Thus, there is a need to develop a unique taxonomy for spatio-temporal game data visualization that is better able to capture the cognitive processes that occur when players examine gameplay context and deduce player reasoning in a spatio-temporal analysis context.

The study presented here sought to address the following research question: "What are the interactive activities and cognitive processes that players engage in when they are analyzing spatio-temporal gameplay data?" We specifically chose to focus on spatio-temporal game data visualizations due to the significant role they play in providing players with the context needed to deduce reasoning, study decision making, and learn from the play of others' $[1,28,66]$. To answer our research question, we ran a qualitative, think-aloud study with seven experienced players of the game Defense of the Ancients 2 (DotA 2) (Valve 2013). Participants were tasked with using the visualization system Stratmapper [2,24] to interact with telemetry data from several gameplay moments of varying length and complexity from two different DotA 2 matches. They were asked to think aloud as they analyzed the data, identify moments of interest, and describe what they though was happening based on what they could see. Audio and screen video from think-aloud sessions were recorded and analyzed via a qualitative thematic analysis procedure focusing on how people interacted with the data and the types of cognitive processes that could be inferred from their think-aloud.

The result is the beginnings of a taxonomy for interaction and sense making in the domain of spatio-temporal game data visualization. The taxonomy, in its current state, consists of seven activities that users engage in while interacting with game data, organized into three categories: Data Interaction (consisting of "study positioning to construct context", "study movement to infer decisions", and "seek details to support sense-making"), Sense Making (consisting of "leverage domain knowledge to fill gaps", "form a hypothesis of context and behavior that evolves over time", "pinpoint events to frame understanding"), and Validation (consisting of "review events to confirm hypothesis"). In addition to identifying these activities, we discuss how they compliment each other and the process by which users move between them as they work to make meaning from the data. We discuss how these findings relate and connect to existing literature on interaction with information visualization. Further, we discuss design implications for future systems presenting this work as a first step towards the development of a full taxonomy for spatio-temporal game data visualization.

\section{RELATED WORK}

In this section we discuss existing work in information visualization interaction taxonomies and spatio-temporal game data visualization. We discuss both topics in terms of how they relate to and motivate the need to create an interaction taxonomy for spatio-temporal game data visualization.

\subsection{Information Visualization Taxonomies}

Within the field of information visualization (infovis), a great number of taxonomies and frameworks have been proposed to formalize visualization design and user interaction [15, 55, 55, 71]. Shneiderman's [58] "Task by Data Type" taxonomy is one of the key examples, which identified seven behavioral tasks that users engage in when exploring visualized data. These were: overview (gain an overview of the entire collection), zoom (zoom in on items of interest), filter (filter out uninteresting items), details on demand (select an item or group and get details when needed), relate (view relationships among items), history (keep a history of actions to support undo, replay, and progressive refinement), and extract (allow extraction of sub-collections and the query parameters)

Proc. ACM Hum.-Comput. Interact., Vol. 5, No. CHI PLAY, Article 260. Publication date: September 2021. 
[58]. Shneiderman's taxonomy is foundational to the field, and many visualization systems and more recent taxonomies explicitly or implicitly referenced it in their development.

However, while numerous taxonomies exist to formalize interaction within information visualization, the research area suffers from two notable weaknesses. First, very little work looks at the cognitive processes involved in making meaning through interaction with data $[47,56]$. This is an important gap in the literature, as these cognitive processes are key to understanding how users turn what they can see in the visualization into actionable knowledge or insights. Examples of taxonomies that do include cognitive elements include Yi et al. [72], Yalcin et al. [70], Patterson et al. [41], and Valiati et al. [61] who focused their taxonomies specifically on these cognitive processes. They included activities such as planning interaction and analysis [70], inferring [61], and managing mental models [72]. However, with the exception of Valiati et al.'s work, which validated the framework with a user study [61], these are based primarily on literature reviews of existing taxonomies, rather than observation of users.

There do exist several visual analytics frameworks that take a more human-centric approach to interaction with visualization, and thus more prominently account for the cognitive elements involved in making sense from data [14, 43]. Demonstrated by the argument presented by Endert et al. [14], there is a school of thought within visual analytics that emphasizes the importance of cognitive processes, such as sense-making, as a part of a human centric approach to understanding and designing for interaction with visualized data. Their "human is the loop" approach to visual analytics aims to go beyond the interactive visualization of information, to better understand how human analysts make sense of data. A concrete example of work within this particular domain is the Knowledge Generation Model for Visual Analytics presented by Sacha et al. [53]. This model, while not presented explicitly as an interaction taxonomy, includes cognitive elements, such as the formation of hypotheses and confirmation of those hypotheses through insights [53]. However, like the interaction taxonomies described above, this model, and Endert et al.'s work, is predominantly based on theory and existing literature, and while a case study demonstrates its functionality, it is not based on a user study.

The notable lack of user studies is the second weakness of existing interaction taxonomy work, which instead favors systematic reviews of existing visualizations and taxonomies. Notable examples of interaction taxonomies based on user studies include the work of Ziemkiewicz et al. [73], who studied how immunologists used data visualization in their lab work, and identified two main strategies (within graphs and between graphs), and Lee et al. [30], who derived the model of novice's information visualization sense-making (NOVIS) from a user study examining how people made sense of unfamiliar visualizations. However, the application areas for these studies are specific to the domain and population, and therefore are not easily generalizable.

Because of these two drawbacks, which are often acknowledged and used to motivate new taxonomy work, there is little consensus among the infovis literature regarding what elements a taxonomy should include. As a result, it is difficult to identify a single taxonomy that is appropriate to adapt to the domain of spatio-temporal game data. Further, much of the existing work focuses on visualizations of aggregate data, which may be applicable to aggregate game data visualizations, but not necessarily transferable to the granularity of spatio-temporal visualizations. As Rodrigues and Figueiras [50] demonstrated, applying the existing taxonomies to spatio-temporal visualizations can present a challenge, as it requires constructs to be reconfigured to account for the more granular data. This suggests that the best approach would be for the domain of spatio-temporal game data to develop its own taxonomy, a stance further supported by the unique characteristics that set game data apart from other data types. 


\subsection{Spatio-Temporal Game Data Visualization}

Game data has become increasingly relevant in recent years, as game analytics techniques gain considerable popularity in the games industry [13]. Popular analytics methods often involve applying machine learning $[9,12,39,46,52]$, statistics [10, 11, 44], or data mining [6] techniques to large gameplay data-sets in order to predict victory or classify players based on notable patterns. Data analytics remains one of the most efficient ways to collect large scale, generalizable insights regarding what players do in-game [24]. For this reason, there is a notable movement that seeks to make the analysis of granular telemetry data more transparent and accessible to human examination through data visualization $[13,35,57]$.

There exist many approaches to game data visualization [35, 65], such as the node link graph developed by Nguyen et al. [37], which clustered players based on their sequences of actions. However, spatio-temporal visualization has grown notably prominent, due to the ease with which it allows human analysts to make meaning from telemetry data by presenting low level, granular, and context sensitive information [2, 16, 24, 36, 64, 65, 67]. One example is the work of Moura et al. [36], who proposed a system for analysts to interact with data superimposed atop the game map, in an approach that, they argued, better allowed one to analyze cause and effect than traditional heat maps.

User evaluations of spatio-temporal data visualization systems have generally shown positive reactions from users $[1,19,28,62,63,66,67]$ leading to further development and evaluation. For example, Wallner et al. [63] and Halabi et al. [19] conducted studies that engaged professional game developers in evaluating spatio-temporal visualizations of player movement in Infinite Mario. Similarly, Wallner and Kriglstein [66] conducted a similar study with players of the online game World of Tanks. In all three studies, the users were able to use the visualizations to understand how players traversed game maps. Further, they were able to provide valuable insights into best practices for visualization design, such as variable opacity being good for at-a-glance analysis [19] or abstracted icons being good for clarity of movement [66].

There also exist interactive, spatio-temporal visualization systems that have shown promise as player-facing tools that can aid in the analysis of play [1,28]. For example, Kuan et al. [28] developed an interactive, spatio-temporal visualization system for Starcraft 2 [3] that synchronizes data presented across maps and timelines. In a user evaluation, they determined that, in situations where the cause of victory or defeat is unclear, their system performs better than existing tools. Participants also preferred their system over existing tools in satisfaction, ease of use, learnability, mental effort, physical effort, time, and helpfulness [28]. Other studies conducted with similar tools have also demonstrated benefits, even when the participants were players who did not possess data analysis backgrounds $[1,18]$.

However, these evaluations focused primarily on judging usability and identifying design best practices. Although some evaluations do include discussions of how users interacted with and used the visualization to complete tasks, it is almost always discussed at a high level, rather than with granular detail [27, 68]. Instead, detailed discussions of how users use, interact with, and make meaning from game data visualizations are largely restricted to theoretical frameworks. One such example is the work of Bowman et al. [4], who suggested a categorization of visualizations for gameplay based on their primary purpose, target audience, temporal usage, visual complexity, and whether or not they were inside the game. Similarly, Medler [34], presented a framework for understanding the functionality of player dossiers within the gameplay experience, suggesting that they give the player an opportunity to learn from their data, which in turn motivates them to keep playing. However, neither framework specifically addressed spatio-temporal data, or examines player interaction with such data at a granular level. 
The work of Penney et al. [42] is notable here in that it does present a detailed understanding of how players interact with information in a granular manner. Based on information foraging theory, they used a think-aloud protocol to generate a detailed understanding of what information StarCraft players wanted from a replay, the paths they followed to find it, and the decision points during play they considered to be most critical. To our knowledge, this is the only work that examines how players explore and make sense of data in such a granular manner. However, this work was motivated by a desire to understand the needs of real time strategy players with regards to understanding the actions of an artificial intelligence (AI), in order to design better explainable AI. Thus, the "data" used in the study is a match replay, rather than a data visualization, and the goal was to understand players' needs in terms of understanding an AI's behavior [42].

As spatio-temporal visualization systems, many of which include interactive components [2, 24, $36,62]$, become more popular within the domain of game data, there is a need for a formal, granular understanding of how users will interact with and make meaning from the data. Specifically, there is a need to develop a taxonomy that describes the activities engaged by players when they interact with a tool in order to make meaning from spatio-temporal game data. Such a taxonomy can better inform the design of future systems by providing designers with a list of user activities that should be accounted for in design.

\section{METHODOLOGY}

To address this gap, we conducted a qualitative, think-aloud [31] study in which DotA 2 players used Stratmapper, an interactive spatio-temporal visualization system, to interact with unfamiliar, i.e. not their own, gameplay data and complete tasks. We conducted a qualitative analysis of the think-aloud results to develop a starting point for a taxonomy for user interaction and sense making in the context of spatio-temporal gameplay data. We specifically chose to develop a unique taxonomy rather than adapting an existing one due to the aforementioned lack of consensus among the infovis literature.

Due to the COVID19 quarantine, all study sessions were conducted remotely over Google Meet, with participants sharing their computer screen while audio and screen were recorded. Institutional IRB approved the protocol and informed consent was given at the beginning of each think-aloud session.

\subsection{Recruitment}

Seven DotA 2 players (whose experience ranged from 3 to 8 years) were recruited via email, social media, and word of mouth from collegiate esports clubs and through snowball sampling. Participants were required to be (1) 18 years of age or older, (2) able to communicate in written and spoken English, (3) able to join a Google Meet call, and (4) in possession of enough experience with DotA 2 to identify and describe basic strategic behaviors. There were no requirements regarding in-game ranking. Aside from years of experience, no other demographic data was collected to avoid biasing analysis towards differences based on age or gender.

Based on previous qualitative studies examining game data visualization [19, 63], recruitment was performed in an ongoing manner and participants were interviewed as they came in. We sought to recruit up to 10 participants for the study, but kept note of data saturation as we conducted think-alouds. After every think-aloud session, the research team discussed the main takeaways from the session and informally noted any new or prominent interaction patterns they observed. After the fifth session, no new patterns were noted. After two more sessions without new patterns emerging, it was determined that the data had reached saturation, and the decision was made to close recruitment at seven participants. 


\subsection{Defense of the Ancients 2}

A game of DotA 2 consists of two teams, the "Radiant" and the "Dire", both consisting of 5 players, who compete against each other in a capture-the-flag style competition. Each team has a base, located on opposite ends of the game map, containing an entity known as the "Ancient". Each player in a DotA 2 match controls an avatar called a "hero", and each hero fulfills a certain role, i.e. "carry", "support", "tank", etc..., within the team. The goal of the game is to protect one's own Ancient while simultaneously trying to destroy the opposing team's. The game map is organized into three lanes, which house towers that fire at enemy players, with forested areas between them, known as the jungles. Both of the lanes and the jungles are populated by various non-player characters that can be slain for experience and gold. Victory is won by killing enemy heroes, destroying enemy towers, and advancing across the map to the opposing team's base.

We chose to use DotA 2 due to the game's popularity, which makes it one of the top esports in the world [60], and due to the availability and accessibility of DotA 2 data. Further, DotA 2 and its predecessor DotA are two foundational examples of the Multiplayer Online Battle Arena (MOBA) genre, which is one of the most popular and lucrative genres in esports. DotA 2 served as the template upon which other popular esport games including League of Legends and Heroes of the Storm are based and thus findings related to DotA 2 data may be easily generalizable to other MOBA games. DotA 2's map, and player positioning upon that map, are also key elements of gameplay, which makes DotA 2 data well suited to spatio-temporal visualization.

\subsection{Visualization Setup}

Stratmapper is a spatio-temporal visualization system that displays raw, low level spatio-temporal game data as event icons superimposed atop the game map, see Figure 1. Stratmapper's interactive components are similar to those included in other interactive spatio-temporal visualization systems for games [1,28], and thus it is representative of the state of the art. Stratmapper's interface also features an interactive timeline that marks what events have occurred, when they occurred, using the same icons as the map. The map and timeline are synchronized such that what is displayed on the map is dependent on what part of the timeline is highlighted. Data points move on the map as the user moves the highlight on the timeline, allowing users to move forward and backward in time and see how events progressed spatially. This allows users to see when and where during gameplay each event was recorded and essentially allows users to "watch" a replay of the game, without actually watching one, and with the ability to stop and examine data in a static manner. Hovering the mouse over action icons brings up tool-tips with more information regarding individual actions and the relevant players. Stratmapper also features a labeling system, see Figure 3, which allows users to highlight sets of gameplay events on the timeline and apply a label, which can be viewed by other users of the tool or exported to another system for analysis. Further details on the functionality of Stratmapper are available in previous work [2, 24].

Data from two past DotA 2 games from a professional league were chosen for use in this study. We chose to use the data of others for three reasons. First, we wanted to observe cognitive processes of extracting meaning from the data, and felt this would be more apparent if the user did not already know the gameplay context behind the data, as they would have with their own data. Second, we wanted all participants to observe the same set of events, which would not be possible with their own gameplay data as the participants were not affiliated with one another. Third, as we discussed in the introduction, interaction with game data includes interaction with the data of others' with the goal of learning from their play. This practice requires granular, context sensitive presentation of data such that users can reason about the causal relationships within the data, and has been used to motivate the development of player-facing spatio-temporal visualizations for game data 


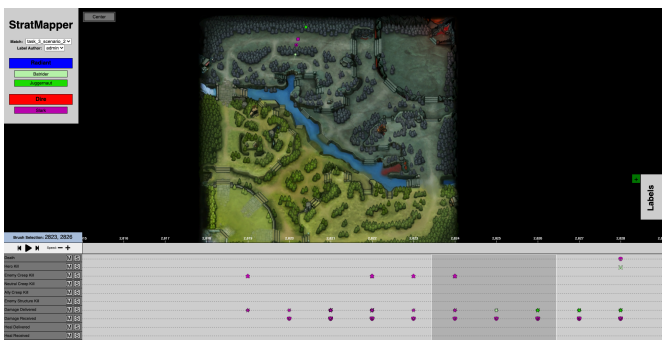

(a)

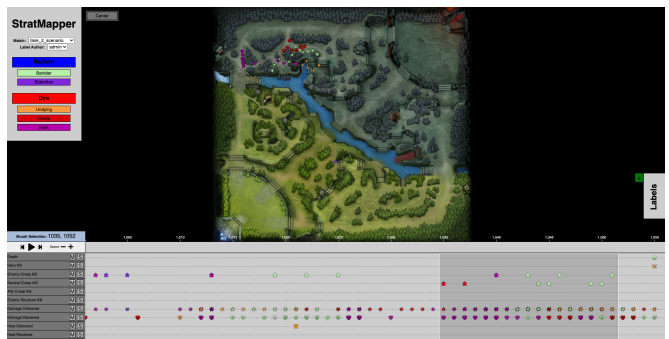

(b)

Fig. 1. The Stratmapper interface used in the study, depicting the localized event from task 2 (left) and the larger event from task 1 (right). At the bottom of the interface is the timeline, where the darker grey highlight is used to determined what data points are seen on the map (only the points encompassed by the highlight on the timeline will appear on the map). On the left is a list of heroes involved in the respective event. Clicking a hero's name in this list will mute their data points. On the left side of the timeline are buttons to mute data points for certain gameplay actions. Above the hero names is a drop down menu that can be used to change the visualized data set.

$[66,67]$ including interactive systems $[1,18,28]$. We recognize this context as one that separates interaction with game data visualization from InfoVis at large, where the in-depth analysis of others' granular behavioral data is less emphasized. Thus, it is critical that an interaction taxonomy for spatio-temporal game data visualization can capture interaction in this context.

\subsection{Think-Aloud Protocol}

Upon giving informed consent, every participant was shown the same Stratmapper instructional video. After watching the video, participants were allowed to ask the lead researcher any questions about the tool or what they would be doing. Once the participant was ready, the lead researcher gave them a link to a web-hosted instance of Stratmapper. The participant was then asked to share their screen and the researcher began recording the session. Before beginning the think-aloud tasks, each participant was given an opportunity to practice using the tool and ask any clarifying questions regarding the visualization or the interface. Each think-aloud session consisted of three tasks that sought to answer the question: "What are the interactive activities and cognitive processes that players engage in when they are analyzing spatio-temporal gameplay data?" Participants were given instructions on how to think aloud before beginning the tasks.

The researchers identified two types of events that an expert looking at spatio-temporal gameplay data would encounter, which required different actions to extract meaning. The first type of event is small and localized, involving few players and occurring over a short period of time. Making meaning from this type of event is expected to involve more cognitive processes, as the analyst must construct the surrounding context themselves, and less interaction with the interface as there is less observable data to examine. The second type of event is a longer event consisting of a series of the smaller, localized events. This type of event occurs over a longer period of time, involves more players, and more data points. Making meaning from this type of event is expected to involve more interaction with the interface, as there is more data to examine and thus there will likely be more scrolling, filtering, and zooming. Similarly, it is expected to involve less cognitive processes as more context is represented by the data.

To capture both scenarios, we created two types of tasks. In one type, the participant was presented with a small, localized event and asked to recognize what was happening. We refer to 
this as a recognition task. In the other type, the participant was presented with a larger event and asked to identify three to five localized events. We refer to this as an identification task.

To prevent cognitive overload, and to ensure that all participants would be observing the same data, the research team pre-selected the events for participants to interact with. One researcher with extensive knowledge of DotA 2 and similar games used Stratmapper to study the two DotA 2 games in detail, and selected three localized events and two longer events to show participants. These events were selected on the criteria that they contained enough interesting and complex gameplay maneuvers to be worth analyzing. Such maneuvers included behaviors such as ganks, team fights, farming, and kills, identified as noteworthy strategic behaviors in previous work [2, 24]

Within Stratmapper, the data for each event was visualized separately, and users could switch between moments using a drop down menu on the left. Only data points related to the relevant actions and players were present for each event. For example, if the event involved three players, only those players would be present in the visualized data. The two longer events featured five and ten players between the two teams, respectively, and consisted of several smaller, localized events. The three localized events featured three players. Two additional researchers with DotA 2 knowledge and experience reviewed the selected moments and confirmed they were appropriate for use in the study.

The five events were organized into five think-aloud tasks as follows:

- TASK 1 - IDENTIFICATION TASK: The participant was presented with a longer event that involved five heroes (three from one team and two from the other). The researchers had identified a sequence of localized events that included farming, jungling, and ganking, located in the top lane and top jungle. The participant was prompted to find three to five gameplay maneuvers of their choosing in the visualization and describe them in terms of the actions, behaviors, and goals involved. While participants were asked to find three to five events, this was not a strict rule, and some participants found less or more than the requested amount.

- TASK 2 - RECOGNITION TASK: The participant was presented with a short, localized event that involved three heroes (two from one team and one from the other). The researchers had identified this event as a two-sided gank in the top lane. The participant were prompted to describe what event the data was depicting, again, in terms of the actions, behaviors, and goals involved.

- TASK 3 - RECOGNITION TASK: The participant was presented with a different short, localized event that again involved three heroes (two from one team and one from the other). The researchers had identified this event as the end of a push in the top lane, when the attacking heroes teleported to safety. The participant was again prompted to describe what event the data was depicting, again, in terms of the actions, behaviors, and goals involved.

- TASK 4 - RECOGNITION TASK: The participant was presented with a third short, localized event that again involved three heroes (two from one team and one from the other). The researchers had identified this event as a gank in the middle lane, where the target got away. The participant was again prompted to describe what event the data was depicting, again, in terms of the actions, behaviors, and goals involved.

- TASK 5 - IDENTIFICATION TASK: The participant was presented with a second longer event, this time involving all ten heroes on both teams. The researchers had identified this longer event as a series of localized events including skirmishes between enemy heroes, jungling, roaming, farming, pushing, and team fights. The participant was again prompted to find three to five gameplay maneuvers of their choosing in the visualization and describe them in terms of the actions, behaviors, and goals involved. This time, however, they were also asked to use Stratmapper's labeling system to apply labels to three to five gameplay events in the 
data. The purpose of this was to observe participants as they identified events, but with a persistent indication of what they had found so far (in the form of the labels). They had free rein to label any kind of event they liked. Similar to task 1, the three to five events prompt was not a strict rule, and some participants labeled fewer or more.

For all three tasks, the participant was prompted to load the relevant moment, provided with instructions on what to do (either extract or recognize maneuvers), and given a reminder to think aloud. Participants were then free to interact with the tool however they wished or felt necessary to complete the task. If the participant encountered a technical issue or had a question the lead researcher would help them. Additionally, the lead researcher would prompt the participant to keep thinking out loud if they became quiet. The researchers performed no other interventions during the think-aloud. Participants were allowed to continue interacting with the tool for as long as necessary for each task, and there was no time limit.

The three tasks were followed by a short open ended interview that gauged how the participant felt about their interaction with the Stratmapper tool, after which the session was completed. Each session lasted from 30 minutes to one hour and was attended by three members of the research team, one who led the procedures, and two who took notes in the background. All participants were entered into a raffle for a $\$ 150$ gift card as compensation for participation.

\subsection{Data Analysis}

Two researchers used ELAN [38], a tool for labeling videos, to analyze and code the screen and audio recordings of the think-aloud sessions. The two researchers followed a thematic analysis protocol $[17,54]$, specifically focusing on how participants were interacting with the data, as observed from the videos, and the types of cognitive processes that the participants engaged in, as inferred by the researchers from what the participants stated aloud. First, both researchers, separately, reviewed a subset of the data consisting of two videos to develop initial lists of behavioral labels. They then reconvened and synthesized a combined list of labels through discussion and comparison of their individual lists, specifically looking at overlaps and differences. They then separated again and individually applied labels from the combined list to $30 \%$ of the data set [5], for an interrater reliability check. The resulting score, calculated using Cohen's kappa [8] was .68, indicating moderate agreement [29]. In the interest of ensuring strong validity, the researchers discussed disagreements and adjusted the combined list by collapsing overlapping labels and redefining those that lacked clarity. They then conducted a second round of inter-rater reliability coding with the refined list that resulted in a kappa value of .82, indicating very strong agreement [29]. The remaining data set was then labeled using the final list.

\section{RESULTS}

The final list of labels represents the physical and cognitive activities engaged by the study participants and presents a foundational understanding of how users interact with spatio-temporal game data visualization in order to develop an understanding of unfamiliar data. Organized into three categories, Data Interaction, Sense Making, and Validation, we present this as the first step towards a formal taxonomy for interaction within the domain. This taxonomy can be seen in Table 1. Further, by analyzing overlaps in label application between the categories, we identify how the different activities in the taxonomy complement each other, and present an initial process model, see Figure 5, for interaction and sense making in the domain of spatio-temporal game data. 


\begin{tabular}{|c|c|}
\hline Activity & Definition \\
\hline \multicolumn{2}{|l|}{ Data Interaction } \\
\hline $\begin{array}{l}\text { Study Positioning to } \\
\text { Construct Context } \\
* * *\end{array}$ & $\begin{array}{l}\text { User studies the positioning of the players } \\
\text { to construct an understanding of the setting for the data. }\end{array}$ \\
\hline $\begin{array}{l}\text { Study Movement to } \\
\text { Infer Decisions } \\
* *\end{array}$ & $\begin{array}{l}\text { User examines movement over time and } \\
\text { infers decisions based on their movement. }\end{array}$ \\
\hline $\begin{array}{l}\text { Seek Details to } \\
\text { Support Sense-Making } \\
*\end{array}$ & $\begin{array}{c}\text { User examines low level actions in a focused and granular } \\
\text { manner to obtain more context in order to explain or } \\
\text { answer questions about data-points and make sense of } \\
\text { the observations. }\end{array}$ \\
\hline \multicolumn{2}{|l|}{ Sense Making } \\
\hline $\begin{array}{l}\text { Leverage Domain Knowledge } \\
\text { to Fill Gaps } \\
* * *\end{array}$ & $\begin{array}{l}\text { User uses knowledge of the game to fill in gaps } \\
\text { in the data to construct context } \\
\text { or infer decisions without additional interaction. }\end{array}$ \\
\hline $\begin{array}{l}\text { Pinpoint Events to } \\
\text { Frame Understanding } \\
\qquad * * * *\end{array}$ & $\begin{array}{l}\text { User identifies events of interest in order to } \\
\text { build a frame for their understanding of gameplay. }\end{array}$ \\
\hline $\begin{array}{l}\text { Form a Hypothesis based on } \\
\text { Context and Behavior } \\
\text { that Evolves over Time } \\
\end{array}$ & $\begin{array}{l}\text { User provides or updates an explanation for } \\
\text { what they believe is happening during gameplay } \\
\text { as represented by the data. }\end{array}$ \\
\hline \multicolumn{2}{|l|}{ Validation } \\
\hline $\begin{array}{l}\text { Review Events to } \\
\text { Confirm Hypotheses } \\
\qquad * * * *\end{array}$ & $\begin{array}{l}\text { User checks for errors in understanding before completing } \\
\text { analysis by ensuring that pinpointed events } \\
\text { conform with the chosen hypothesis. }\end{array}$ \\
\hline
\end{tabular}

Table 1. The taxonomy of user interaction for spatio-temporal game data visualization, consisting of seven activities organized across three categories. The symbols next to each activity name mean the following: * means that this activity applies to all visualization, ${ }^{* *}$ means that this activity applies to all spatio-temporal visualization, ${ }^{* * *}$ means that this activity applies specifically to visualizations for game data, ${ }^{* * * *}$ means that this activity applies specifically to spatio-temporal visualizations for games.

\subsection{Data Interaction}

The three activities in this category relate to how users would use the tool to read the data. This is distinct from the next category as these activities emphasize observable interactions with the tool. While these activities were used to support cognitive processes, these relate specifically to what information the user would focus on acquiring as they attempted to understand the game data, and how they would use the tool to facilitate the extraction of that information.

4.1.1 Study Positioning to Construct Context. The first activity in this category refers to the process in which a user examines the location of players on the map in order to construct a mental model of gameplay context. This activity involved examination of stationary data points, representing gameplay actions, in terms of where they were located on the map, which indicated where a player was, and could be used to infer information about the context of play, i.e. which team was 


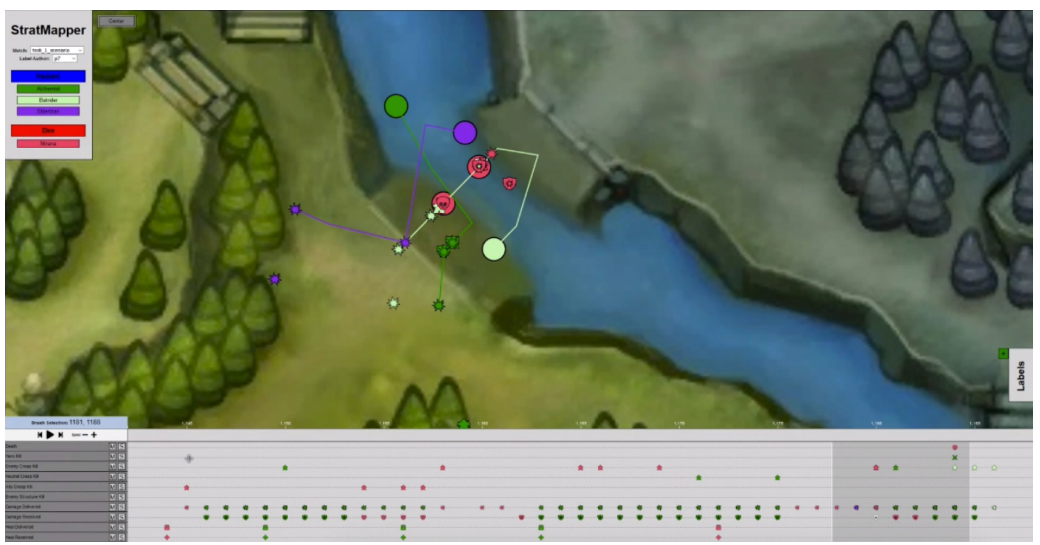

Fig. 2. While studying positioning, participant 7 zoomed in on the middle lane area of the map, where the players of interest were located.

performing better or what point in gameplay the data was drawn from. While engaging this activity, the participant would make statements regarding the positioning of players and how this impacts their understanding or construction of a gameplay context. For example: "my guess is that both the Silencer and the Juggernaut here were pushing the creep wave here to attempt to break the tier three towers" (Participant 2) This illustrates a moment during analysis in which the participant noticed that two Radiant side heroes were deep into the Dire side of the map and was able to make a judgement regarding the context of the game based on this positioning. Participants would also comment on positioning of players in relation to actions, sometimes pointing out how these were odd or defied their understanding of context. For example: "it doesn't make sense why Batrider is taking so much damage since he's closer to his side of the map" (Participant 4). This illustrates a moment during analysis in which the participant was confused about gameplay context because they thought the presence of certain actions in relation to a player's position did not make sense. While studying positioning, participants would sometimes zoom in on an area of interest on the map for a closer analysis of the players' positions. An example of this can be seen in Figure 2 .

According to the labeling, studying positioning comprised $20-40 \%$ of each participants activities within the data interaction category, with the exception of participant 2 , for whom it only comprised $5 \%$ of their data interaction activities. Studying positioning is not, itself, unique to game data, but rather is an interaction pattern expected of, encouraged, and afforded by spatio-temporal visualization systems. What is unique to games, however, is the important role that studying positioning plays in building a mental model of the gameplay context. As the utterances above demonstrate, by studying positioning, participants were making themselves aware of the spatial context of the game and seeking out situations, such as unusual positions, that warranted further analysis.

4.1.2 Study Movement to Infer Decisions. The second activity in this category refers to the process in which a user used the tool to examine how players moved over time and infer players' decisions based on that movement. Participants would use Stratmapper's timeline feature to "scrub" backwards and forwards in time, which would cause the data points on the map to shift position based on what actions were taken at the selected time and where, allowing them to see a player's movement. Movement was sometimes studied in a holistic manner, with movement across the entire moment examined in one scrub through from beginning to end, and in a group manner, with multiple 
players visible simultaneously. Participant 7 is an illustrative example of this, who zoomed out enough to see the entire map and scrubbed through the entire moment, verbalizing their intent as: "I'm just going to look at an overview just to get an idea of what's happening." At other times, movement was examined more granularly, focusing either on smaller events within a moment, or on a single player with other players being ignored or filtered out, to better understand the moment to moment decisions that were made. Examples of what participants would say regarding player decisions when studying movement are "...he rotates to the top lane through the river, turns back around for a second, but he keeps going" (Participant 1) and "you can see over here the Templar is chasing and hoping to dive the tower" (Participant 4).

According to the labeling, for each participant, movement comprised $40-60 \%$ of their activities within the data interaction category, making it the most prominent of the three activities. Similar to studying positioning, studying movement is not inherently unique to games, but an interaction afforded by spatio-temporal visualization and present in most contexts in which such an approach is used to visualize data. That being said studying movement played a key role in uncovering player decisions. Specifically, as the utterances demonstrate, participants would study movement to observe what players did and identify or infer their decisions, such as how participant 4 used the movement of the player to identify their decision to dive the tower.

4.1.3 Seek Details to Support Sense-Making. The third activity in this category refers to the process in which a user would intentionally seek out more information regarding the state of the game, such as the items held or the health of a given hero, in order to support the process of making sense of the data. In a generalizable sense, seeking details is the act of seeking out low level information that was not immediately visible from the tool, but which provided information regarding the state of the game. In the specific case of Stratmapper, detail seeking manifested through interaction with the system's tool tips. Specifically, participants would trigger tool tips by hovering over icons in order to read detailed information regarding health, damage dealt, or who the target of a skill was. An example of a participant interacting with the tool tips to seek details can be seen in Figure 3(a).

According to the labeling, the extent to which an individual participant would engage in seeking details ranged from $20-40 \%$ of data interaction activity. Similar to the other actions in this category, seeking details is not unique to game data, and is, in fact, a staple interaction of all types of data visualization [58], where it is also often used to support sense-making, and thus is not unique to games nor to spatio-temporal visualizations. However, we did observe two patterns regarding how detail seeking was used in line with the cognitive processes involved in building a mental model of play.

In the first pattern, details were sought to answer a question and detail seeking often came after the other activities in this category. For example, after engaging activities in the data interaction category, participant 6 noticed that one hero was healing and brought up the tool-tip to examine the hero's held items. When they found the answer they were looking for, they stated it out loud, saying "tranquil boots [a held item that heals over time], ok". In the second pattern, details were sought to support construction of context detail seeking often came before the other activities in this category. For example, before engaging in data interaction activities in earnest, participant 5 wanted to know when during the game the data points occurred, and brought up the tool-tip to see the time stamp. They stated this goal out-loud, saying "let's see, just to get a memory on the time stamp here...ok so a little bit later in the game". In both cases, seeking details supported the ongoing process of making sense of the data. 


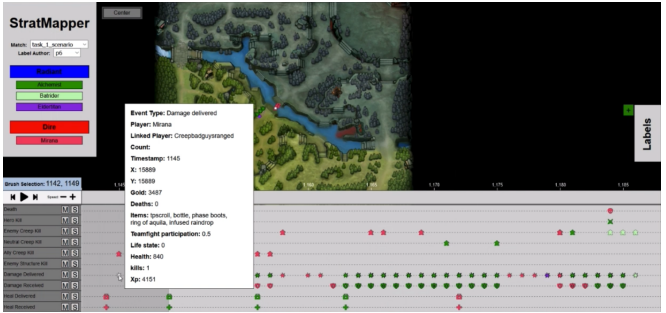

(a)

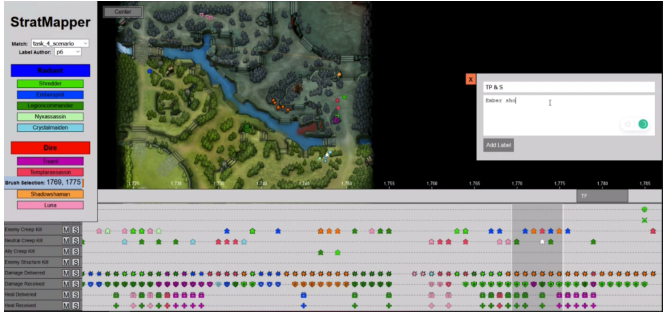

(b)

Fig. 3. An example of participant 6 using a tool-tip to seek details (left) and applying a label to the data during task 3 (right).

\subsection{Sense Making}

The three activities in this category relate to the cognitive activities that users would engage in while interacting with the data in order extract meaning and develop an understanding of gameplay. These are distinct from the previous category in that they do not connect to observable actions but rather they are cognitive processes engaged by the participants, which were inferred by the researchers based on think-aloud vocalization.

4.2.1 Leverage Domain Knowledge to Fill Gaps. The first activity in this category refers to the process by which participants would fill in gaps in information based on their own knowledge of the game. With regards to gaps in information, there were two kinds of scenarios identified. In the first scenario, the data did not include all information regarding what players were doing, however, participants were able to use domain knowledge to fill in the missing information. For example, Stratmapper does not show ability usage, however, participants were able to leverage domain knowledge to infer ability usage based on observable information, i.e. "it looks like he surges [ability] away because he's running really fast" (Participant 3 ).

In the second scenario, information was included, however, participants did not need to look at it to understand what was happening. For example, participant 1 was able to recognize that a player was farming a jungle camp based on their movement and did not need to look at who the target of the player's attacks were, which was included within a tool-tip. Participant 1 vocalized this during their think-aloud, stating: "when Oracle is pulling this creep camp here, you would have to read into it and you would see that that is what's happening because he's delivering damage to neutral camps, but I did not do that, I just assumed he was pulling based on his movements". Similarly, participant 5 recognized that a teleport was going to happen based on the positioning of a player and without having to check the items for a teleport scroll. They stated: "I already know that this is a pretty common, even in the modern updates of DotA 2, this is a pretty common spot to want to get away from someone, there's not a lot of vision in this area."

Domain knowledge was also leveraged to explain why certain patterns or details existed in the data. For example, participant 6 noticed, while examining tool tips, that a hero's health was increasing. After looking at the items the hero held, they were able to explain this pattern with domain knowledge, stating: "tranquil boots [an item held by the hero in question] gives heal over time". At a higher level, all participants noticed a pattern in which data points for one hero, Legion Commander, were predominantly located in the jungles. All participants commented that farming the jungle was a common strategy for the hero, effectively leveraging their domain knowledge of popular gameplay strategies to explain an observable pattern in the data. 
Based on label application, leveraging domain knowledge ranged from $20-40 \%$ of sense making activities for each participant. Domain knowledge is not emphasized in existing taxonomy work, as much of the literature assumes novice users who are not familiar with the domain the data comes from. By contrast, using data to learn in the context of play requires enough domain knowledge to recognize game states (such as a given player possessing a given item) and the causal relationships between those states and the player's actions (such as the player using that item to heal). Thus we recognize leveraging domain knowledge as an activity specific to game data visualization, though not necessarily restricted to spatio-temporal game data visualization.

4.2.2 Pinpoint Events to Frame Understanding. The second activity in this category refers to the process by which participants would identify or single out an event that occurred within the moment and use it to frame their understanding of gameplay. These events were often pinpointed with the aid of Stratmapper's action icons. In some cases, these events were identified from a single action. For example, participant 7 pinpointed an event from a single death action, stating "and then there's a death on the Shredder". In other cases, these events were inferred from a collection of actions, often facilitated by an activity from the information interaction category. For example, participant 4 examined a set of stationary action icons at a given map location (studied positioning) and pinpointed a camp pull, stating "Oracle here, being the good support that he is, is pulling a neutral camp". There were also moments where an event could be pinpointed based on player positioning alone, without any action icons present. For example, participant 5 saw Juggernaut's location, during a point in time that the hero was taking no actions, and pinpointed a set up for a gank, stating "Juggernaut is setting up [a gank] in the trees right here". In some cases, a pinpointed event would be the first thing a participant would analyze within a moment. For example, Participant 3 began their analysis of one moment with the following pinpoint: "it looks like Timber is going to die at the end, and no towers go down, and it looks like everyone else is going to be scuffling with some heals thrown in".

Based on label applications, pinpointing events encompassed 15 to $25 \%$ of participants activities in the sense making category, making it the least common activity within the category. The only exception to this was participant 6 , for whom it comprised only $5 \%$ of activities in the sense making category. Pinpointing events is more than picking out an important data point (which is discussed in existing taxonomies) but rather the act of identifying a single or collection of data points as influential to or providing a framing for one's ability to comprehend the rest of the data. As such, pinpointing events is different from what is described in existing taxonomies as it encompasses this cognitive recognition process, which is largely absent from existing work. Thus, this activity is unique to game data visualization. Further, as pinpointing events appears to be exceedingly reliant on the ability to comprehend the context of play, we believe it is unique to spatio-temporal game data visualization, which is able to present enough context to facilitate the activity.

4.2.3 Form a Hypothesis of Context and Behavior that Evolves over Time. The final activity in this category refers to the process by which participants would provide theories for what was happening within the game, in terms of gameplay context or player reasoning or goals, based on what they could observe from the data. Three kinds of hypotheses were observed: hypotheses for what had already happened before the moment began, i.e. "just from the start of it, it looks like they may have already taken the tier two [referring to a tower]" (Participant 5), hypotheses for what had not yet happened but was about to, i.e. "it looks like Batrider is positioning, potentially, to jump that Slark" (Participant 3), and hypotheses for what was currently happening, i.e. "Juggernaut and Silencer, they might be pushing over here" (Participant 6). Participants would also form a hypothesis and then sometimes update it later as they discovered more information. 
As can be noted from the three examples, a hypothesis was defined as a guess regarding game context, player goals, or player reasoning. In other words, it was an inference on the part of the participant regarding information they could not observe. The formation of a hypothesis was facilitated by interaction with the data as well as leveraged domain knowledge, and a hypothesis could then be validated or invalidated by a pinpointed event. For example, participant 5 hypothesized that the hero Juggernaut "just flies over and snags him, I don't think there was a blink dagger", which was concluded by studying movement and leveraging domain knowledge. Specifically they leveraged the knowledge that Juggernaut is able to jump to a target without the aid of an item, and that an item called a blink dagger, which facilitates such movement, exists. However, participant 5 later pinpoints an event that overturns this hypothesis: "yep, ok, so he did blink in." Specifically, participant 5 was seeking details to confirm their hypothesis, saw the blink dagger in Juggernaut's items list, and was then able to pinpoint an event (the use of the dagger) that overturned their previous hypothesis.

Based on label application, hypothesis formation ranged from 30 to $60 \%$ of the activities in the sense making category. This makes hypothesis formation the most common activity within sense making, likely because it is the foundation upon which an understanding of events, the primary goal of the category, can be built. Existing taxonomies do discuss hypotheses, however, they are with regards to the data, and what it represents, rather than the gameplay context or the players' decisions and goals. Thus, in the context of game data and this taxonomy, forming a hypothesis is inherently different than in existing taxonomies, due to its focus on comprehending the gameplay itself, rather than the data. We recognize this activity as one that applies to game data visualization, but not one that is restricted to spatio-temporal game data visualization.

\subsection{Validation}

This category consists of a single activity, which is categorized separately as it encompasses the act of checking rather than interacting with data or making sense of it.

4.3.1 Review Events to Confirm Hypotheses. The last activity refers to the process by which participants were observed to validate their understanding of events at the conclusion of an analysis. More specifically, participants were observed to play through the entire scenario again, and would state or narrate what they were observing, and how this supported their hypotheses. If everything they observed during this process supported their hypotheses, they would synthesize them into a single hypothesis for the entire moment. Examples of this can be seen in: "so they gank him from both sides and he seems to die" (Participant 1) and "just a classical jump in and kill the carry" (Participant 5). If they observed data, at this point, that contradicted one of their hypotheses, they would cease the review and re-engage the other activities until they had updated their hypothesis. They would then review again.

Because review occurred only at the end of analysis, it comprised less than $10 \%$ of each participants activity overall according to label applications. In the context of game data, reviewing was not to confirm one's assumptions regarding data presentation, but rather the gameplay itself. This emphasis on gameplay context over data representation sets the action of reviewing in the context of game data visualization, as captured by this taxonomy, apart from the reviewing actions encompassed by existing taxonomies. Thus, we recognize it as an activity unique to game data visualization, and one that is further specific to spatio-temporal visualizations of game data, as the activity relies on the ability to observe granular depictions of events, which is facilitated by spatio-temporal visualization. 


\section{Data Interaction Meaning Making Validation
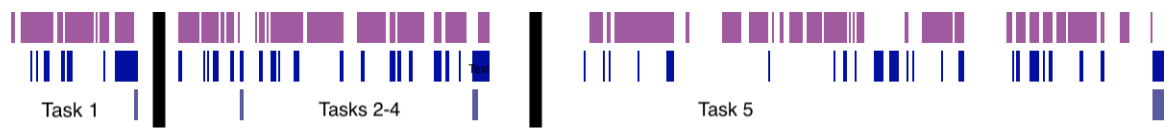

Fig. 4. A plot of label applications for the three categories for all tasks for participant 2, demonstrating overlap between activity types.

\subsection{Overlap and Interplay between Categories}

Examining the label applications for the different action categories, a relationship can be observed between data interaction and sense making. Figure 4 displays a plot of label applications for Participant 2. Each row of colored bars corresponds to a label category (data interaction on top and sense making in the middle), and each colored, vertical bar corresponds to an applied label. The black vertical bars that span all three rows mark the gap between tasks. The plot displays the application density for each label category across the think-aloud session.

It can be observed from this plot that there is a prominent overlap between data interaction activity (top row) and sense making activity (middle row) in that both types of activities appear to happen at the same time, as observed from the pattern in which the colored bars in both rows overlap. This overlap can be seen in the plots for all participants and is the result of an observed interplay between the three categories. Specifically, the activities in the data interaction category facilitated the activities in the sense making category, by permitting the user to find and extract observable information that allowed them to construct meaning. An example of this facilitation can be observed in this excerpt from participant 3's session, labeled as "studying positioning" within the data interaction category and "forming a hypothesis" within the sense making category: "I would assume Radiant has a pretty good tower advantage if he's that deep in". In this moment, the participant was studying the positioning of the players at the beginning of the moment in order to construct an understanding of gameplay context. This allowed them to formulate a hypothesis regarding the context of the game, specifically, that one team had a significant lead on the other team.

At the same time, however, the activities in the sense making category motivate further activity in the data interaction category, by encouraging players to look for information that confirms theories or fills holes in the understanding of events. An example of this can be observed in participant 2: first, this participant is observed to be studying the movement of a hero and inferring their decisions. While examining how the hero appears to teleport back to their team's base, participant 2 states "so they get away from Slark who comes to stop them from pushing top lane". This statement was labeled as "studying movement" with regards to data interaction, and "pinpointing an event" with regards to sense making, as the studying of the hero's movement was what allowed the participant to recognize the event (the teleport), which has allowed them to then frame their understanding of what happened. Participant 2 then goes on to begin looking at tool-tips, stating "it's probably a spin-TP". This is labeled as both "seeking details", as they are looking for information in a tool-tip that can support their sense-making process, and "forming a hypothesis", as they have presented the theory that the hero in question used a trick called a "spin-TP", which involves using a skill to prevent interruption and then using a teleport scroll. However, in this situation, the sense making activity (forming the hypothesis) is motivating the data interaction activity (seeking the details) in that participant 2 is now looking for information that can confirm their hypothesis that the hero used the spin-TP trick. This is clarified by the participant themselves as they state "I kind of want to know if it was a TP spin", while continuing to look through tool-tips. 
The activities in the data interaction and sense making categories are connected in a cyclical process that facilitates the formation of a mental model, an internal conception of an external phenomenon [51], of what was happening during gameplay. Data interaction allows one to observe details that facilitate sense making, which in turn, motivates further data interaction as users seek out additional information to confirm and expand upon that meaning. It is likely that this cycle in which interactions with an interface prompt cognitive processes which then motivate further interactions exists generally across all data visualization contexts. While some taxonomies do discuss mental models, the details of how these are formed is largely under-represented in taxonomy literature due to the aforementioned lack of emphasis on cognitive processes. A detail that is unique to game data visualization, however, is this emphasis on creating a mental model of gameplay context, whereas existing taxonomies only discuss mental models in the context of comprehending the visualized data. Also worth noting from Figure 4 is the greater density of data interaction activities compared to sense making activities. This is due to the fact that a user may need to identify a number of observable details through data interaction before they are able to make meaning of the events.

Similarly, reviewing (the only activity in the validation category in the bottom row) has the lowest density on the plot in Figure 4, and typically comes at the end of a task when analysis of the moment was complete. As discussed previously, reviewing was identified as an act of checking one's assumptions for errors by looking for missed details and summarizing the observed event as it is understood in a concise manner. It therefore stands to reason that it would occur at the end of analysis, after the mental model has been constructed, to check for flaws.

By examining this interplay between the categories, we identify a preliminary process model, seen in Figure 5, for how users make meaning from spatio-temporal game data in terms of how they engage the seven activities discussed above. Specifically, participants will engage in the data interaction activities of studying positioning to construct context, studying movement to infer decisions, and seeking details to support sense-making interchangeably with the sense making activities of leveraging domain knowledge to fill gaps and pinpointing events to frame understanding. These activities are part of a larger process of building an understanding of gameplay context and player behavior from the data. That understanding is then used to form a hypothesis regarding context and behavior, which may evolve over time, and may trigger additional executions of the five previously mentioned activities. If the user believes they have enough hypotheses to satisfactorily explain and understand the data, they will proceed to validation and review the events to confirm their hypotheses. If they find a discrepancy, they will then return to the process of building an understanding of context and behavior in order to form a more accurate hypothesis This entire process is part of a larger process of constructing a mental model of gameplay, which is the ultimate result of interacting with the data.

\section{IMPLICATIONS FOR DESIGN AND FUTURE RESEARCH}

In this section we will discuss the implications of the taxonomy on the design and research of future spatio-temporal visualization systems.

\subsection{Positioning and Movement}

It comes as no surprise that the users of a spatio-temporal visualization system would spend the bulk of their time examining the positioning of data points and how that positioning changes over time (movement), as these are what the systems excel at. However, many existing spatio-temporal visualizations systems do not include interactive features and therefore do not facilitate user control over the presentation of movement. Instead, movement is presented in a static manner, with arrows and paths drawn atop the map $[63,66]$. 
Construct a Mental Model of Gameplay

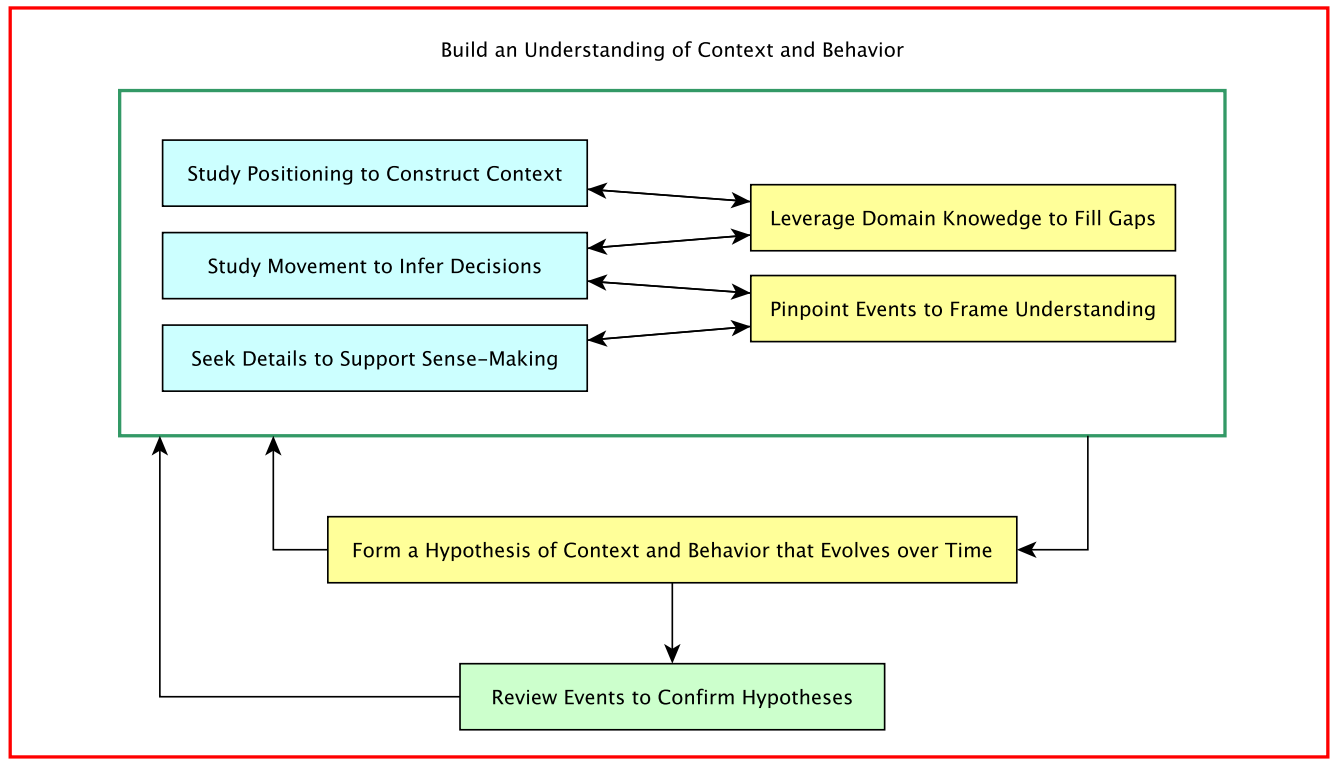

Fig. 5. A preliminary process model to describe how users engage the activities in the taxonomy (see Table 1) in order to understand and extract meaning from spatio-temporal game data.

The results of our study not only highlighted movement as a critical activity within the overall process of understanding and interpreting spatio-temporal gameplay data, but demonstrated the value of allowing users to examine movement in an interactive manner. All participants studied movement by manipulating the timeline selection feature to see how the positioning of each player changed over time. The implication of this finding suggests that future spatio-temporal systems should consider including interactive features that allow users to examine movement in an animated manner. Further, users tended to move time forwards and backwards and did not always go over the entire time frame of the moment, instead focusing on smaller events in an iterative manner. This result suggests that providing users with finite control, rather than a play/pause feature would better serve their needs. This particular implication is likely applicable to all spatio-temporal visualization systems, rather than only those built for games, as there are likely other domains where users would benefit from interaction.

\subsection{Details on Demand vs. Domain Knowledge}

Perhaps the most persistent concept of information visualization taxonomies, details on demand refers to the inclusion of granular, detailed information such that it can be accessed when needed $[15,55,58,70,71]$. All participants during the study engaged in the "seek details" activity at some point, often to confirm their hypotheses for the events they observed. This suggests the continued relevance of the concept, and implies that future systems should continue to adhere to the practice, ensuring that all details are available within the interface and able to be accessed as needed.

However, another notable result that emerged from this study was the significance of domain knowledge, and the extent to which participants did not need to seek details, as their domain 
knowledge meant that they already knew what they were looking at. This emphasizes the importance of the "on demand" part of "details on demand" and implies that future systems can reduce the risk of cognitive load or mental strain by hiding granular details from the main view. This does, however, depend on the audience for the tool. While players are likely to be satisfied using their domain knowledge to interpret events, developers analyzing data in order to make game adjustments would likely want more information up front. Further, those newer to the game, and with less domain knowledge, may require more details up front as well. This suggests that variable arrangements of detailed information should be considered in future system design, depending on the skills, knowledge, and needs of the audience. This implication likely applies to all visualization systems, regardless of domain, and whether spatio-temporal or otherwise.

This also has implications for abstraction of data. While abstraction is a promising avenue for increasing the readability of game data visualization [22], it may interfere with users' ability to trigger domain knowledge. Specifically, Stratmapper features no abstraction of data, instead presenting each data point as what it is, where, and when it occurred. This granular information was recognizable to the participants and triggered their domain knowledge, allowing them to generate an understanding of gameplay events. Abstraction may obscure too much information, and could possibly interfere with this process. Thus, future systems should be cautious of the extent to which data is abstracted, and may want to consider interfaces that include as much contextual detail as possible to facilitate the use of domain knowledge. These implications may be unique to data visualization systems for games, where being able to extract extensive contextual detail is a necessity when using data to learn. It may be further specific to spatio-temporal gameplay visualizations, as other types may suffer from information-overload without abstraction. This question can be explored further in future work.

\subsection{Pinpointing Events and Forming Hypotheses}

Although the participants in this study were experienced DotA 2 players, they were examining data from gameplay that they were not a part of. Thus, a great deal of their ability to extract information from the data hinged on their ability to form a comprehensive hypothesis, which in turn, hinged on their ability to pinpoint events. In fact, these activities are at the core of constructing a mental model, which is the overall process that emerged from the results of the think-aloud study. In infovis literature, mental models are often discussed in terms of how users perceive data or understand the functions of the visualization $[23,32,33,70,71]$. However, here, the emphasis is on users building a mental model of the gameplay context, based on hypotheses of what occurred during gameplay formulated from the events they can pinpoint in the data.

The iterative activities of forming and updating hypotheses are reminiscent of activities included within the NOVIS model proposed by Lee et al. [30]. Specifically describing how people make sense of unfamiliar visualization, the model includes "constructing a frame" to make sense of a visualization and "questioning the frame" when they doubt or need to verify it. In our case, as discussed in results, we saw a similar pattern in how users generated a hypothesis and then sought verification through details and pinpointed events, especially if they came to doubt their hypothesis However, in our case, the hypotheses were related to understanding the gameplay events depicted in the visualization, rather than the visualization itself. Of note however, is that those gameplay events were unfamiliar, just as the visualizations in Lee et al.'s study were unfamiliar [30]. Thus, it may be that the iterative acts of creating, validating, and updating hypotheses are driven more by a need to understand unfamiliar artifacts than any specific domain context, and future research may want to explore this further.

For future systems, the key roles pinpointing events and forming hypotheses play in building a mental model of gameplay suggests opportunities for models or AI assistants that can aid users 
by automating the processes. However, given that user's hypotheses were often updated after being first proposed, the results also emphasize that accurate identification will not be an easy task, and that human users should always be able to change, update, or correct any machine driven identification. Further, the emphasis of these activities also implies the value of tools such as Stratmapper's labeling system, which can allow users to track what they have identified as they continue their analysis, and allow them to better remember their hypotheses when they review. This implication primarily applies to spatio-temporal visualizations for games, where there is a strong emphasis on using pinpointed events to form hypotheses, which are then used to pinpoint more events, in a loop that ends in the generation of a mental model of play. While this may apply to game data visualization more generally, it is difficult to say if other forms of visualizations offer enough contextual detail to form robust hypotheses, and this question can be explored further in future work.

\subsection{Getting the Big Picture vs. Going Granular}

From the results, both the taxonomy and the process model, it is apparent that interacting with spatio-temporal gameplay data involves both looking at the data in a holistic manner as well as a granular one. Specifically, as discussed in the results, participants would often begin by examining positioning and movement of everyone across the map, to get an overview of events. Then, they would get into the details of specific maneuvers, zooming in and focusing on specific players. This resonates with what is discussed in existing taxonomies, which discuss gaining an overview first $[55,58,72]$, then exploring data more granularly by zooming and filtering $[15,70]$. For future systems, this suggests that they should ensure that user's have the ability to see data in both a holistic manner, in which they can see everything at once, and a focused manner, in which they can zoom into an area or player of interest.

When discussing these insights though, it is noteworthy that participants did not always engage in filtering, despite this being an action described extensively in previous work $[55,58,71]$ and a feature supported by the Stratmapper interface [2]. It was because of its inconsistent, and not predominant, use that it was not included as a unique activity within the taxonomy. Those participants who did use Stratmapper's filtering mechanism would only filter players out temporarily, usually to facilitate a more granular study of a given player's movement patterns, and would always bring their data back to the visualization afterwards. Further, no participant ever filtered out any type of event, despite this being a functionality of the tool. This aversion to filter, and tendency to restore the unfiltered data, suggests that participants were reliant on the additional data to make sense of what they were seeing. It may be that participants were inferring contextual information from the additional data points, and were therefore resistant to remove them out of concern that they would miss events that were critical to understanding gameplay. This may be particularly relevant in the context of team based, multiplayer games, as it is often the actions of one player that influence the actions of another. Future work may want to examine this phenomenon in further detail, to examine the extent to which filtered vs. holistic data is able to impact users' understanding of events.

The act of undoing filtering and restoring the previous view within the visualization brings to mind the concept of "history", or the ability to return the visualization to a previous state, discussed in existing taxonomies $[15,55,58]$. However, while previous work discusses this in the context of retracing one's steps or undoing a mistake, here it appears to be more a case of restoring the big picture when one has completed granular analysis. For future systems, this suggests that, although the ability to go granular with analysis must be supported, it must not be done in a way that removes data from the user's view permanently. Any filtering that is facilitated must be able to be removed, such that the context of the game at large, as inferred from the extra data, can be 
restored. This need to restore the big picture is further supported by the "review" activity, which was always enacted with no player filtered out. Thus, it would appear that it requires the presence of all of the data such that the user is able to check their theories and ground the findings of their granular analysis within the context of the gameplay as a whole. These implications, while especially important in the context of game data visualization, are likely applicable to visualization in other domains as well. While users may not always be attempting to confirm a hypothesis or validate a mental model of context, there are likely contexts outside of games in which a user will want to return to the "big picture" of the visualized data.

\section{DISCUSSION}

InfoVis taxonomies do not emphasize the significance of understanding others' decision making processes, goals, or reasoning, whereas the players in our study were driven to build mental models of all of the above, highlighting the need for a specialized taxonomy. Existing taxonomies do discuss the formation of mental models, however, this discussion is always with respect to how users build a mental model of the data in terms of understanding what the data points represent and how to read them. By contrast, the mental models that were being formed by participants during the think-aloud sessions were about the gameplay context, i.e. the circumstances of the game itself, and how that context informed the decisions the players made and the actions they took.

This phenomenon is because, when analyzing others' data in the context of learning and mastering play, it is critically important to be able to understand their reasoning behind each decision, and how it relates to the context of the game. As discussed previously, players will often turn to the gameplay of others, especially those who are more experienced, in order to observe their gameplay and learn new skills, or compare it against their own to identify areas for improvement [69]. However, in order to learn new skills or techniques, explanations regarding reasoning and goals is often necessary to build an understanding of the causal relationships between gameplay context, decisions, and outcomes. In the context of video streams or broadcasts, the player or a commentator can explain what is happening over a voice-over, and help build these causal relationships [7, 20, 26, 59]. Data visualizations, and especially spatio-temporal ones, however, offer a more granular look at gameplay than what is afforded by video [25]. They also allow players to actively draw their own conclusions about others' play, instead of relying on a commentator, which can result in greater learning gains through self-regulated reflection [40]. However, it also requires the users of the visualization to uncover the reasoning and game context themselves, as the subject is not there to explain. Thus, they engage in a series of complex and critically important cognitive processes intended to build a comprehensive mental model of gameplay. These cognitive processes, which are captured by our preliminary taxonomy, are what set interaction with game data visualization apart from interaction with visualization in other domains, and illustrate the need for a specialized taxonomy.

While the contents of the taxonomy may initially appear to be common sense, the formalization of interaction is critically important to ensuring that future spatio-temporal visualization systems include the correct interaction affordances and are able to effectively meet users', especially players', needs. Because there is currently no formal understanding of how players make meaning from visualized data, there is no agreement regarding what types of interaction and analysis processes should be facilitated by systems. For example, many visualizations in existing literature are static images (no interaction) $[19,66,67]$ which will not facilitate the study of movement and may not sufficiently facilitate the study of positioning. In another example, Afonso et al. [1] conducted a user study with VisuaLeage, an interactive, spatio-temporal visualization system similar to Stratmapper, but that operates with data from League of Legends [48]. Their results include three high level techniques for how people analyzed information. These techniques include people who used their 
previous experiences with the game to interpret data beyond what they could explicitly see, and those who would create complex scenarios that were not necessarily verifiable through the app itself [1]. Afonso et al. go on to suggest that designers would want to prevent the derivation of incorrect information, and thus should not promote such analyses. Our taxonomy, however, suggests that these actions are critical to players being able to learn from visualized spatio-temporal data, and that preventing them may be detrimental to the goal of developing visualizations for learning and mastering play. In this way, a formal interaction taxonomy for spatio-temporal game data visualization can better inform the design of future systems, to ensure that they are able to meet players' needs.

\section{LIMITATIONS}

The work presented here is a starting point to developing a formal taxonomy for user interaction with spatio-temporal game data visualization. However, we do acknowledge some limitations of the research. The primary limitation is the use of Stratmapper, a specific spatio-temporal visualization system, with a very specific set of features. While Stratmapper's features do represent the current state of the art for interactive spatio-temporal visualization systems [1,28], we acknowledge that Stratmapper's specific design and features likely biased participants towards certain activities while interacting with the data. To address this bias, additional studies with other spatio-temporal visualization systems, with different features, are necessary to generate a more general understanding of how each activity manifests while interacting with the data. We hope to address this in future work. The work here is meant to present a starting point that future work can build upon in order to generalize and validate the taxonomy.

We also acknowledge that this study examined interaction with spatio-temporal data from only a single game, DotA 2 . We acknowledge that this almost certainly impacted the patterns that we saw. However, we argue that the findings of this work should be generalizable to other MOBA games, such as League of Legends and most likely to any games in which player positioning and movement across a map are critical to gameplay. Further, we argue that those activities within the taxonomy that we identify as relevant to game data visualization beyond spatio-temporal systems may generalize to all games, regardless of whether they include a spatal component. As this is intended to be a starting point for developing a taxonomy, we present our findings as a preliminary taxonomy and process model for interaction with spatio-temporal visualization of MOBA data. A proper study of generalizability and expansion of the taxonomy to encompass other game genres will be explored in future work.

Additionally, we acknowledge that this study had a small sample size with seven participants, and that this is not enough to argue for statistical significance, or to draw conclusions regarding interaction strategies and demographic information. However, as we saw saturation in behavior within our sample, and because this is qualitative work, we argue that the results are still valid. Future work can validate these findings with a larger sample, and explore connections between behaviors and demographic characteristics (such as years of experience).

Finally, we acknowledge that this study specifically examined how participants interacted with and made meaning from gameplay data that was unfamiliar, i.e. not their own and not from a game they were a part of. Thus, we acknowledge that the phenomena we observe are biased by the participants' lack of pre-existing knowledge of the gameplay context, and that the processes used by those examining their own data may differ, especially with regard to constructing mental models. We chose this specific path due to the significant role that spatio-temporal visualizations play in making meaning from the data of others' in the context of learning and mastering play $[1,28,66]$. However, we acknowledge that additional studies that examine participants' interacting 
with their own data are needed to expand and generalize the taxonomy. We hope to address this in future work.

\section{CONCLUSION}

Game analytics is turning increasingly to spatio-temporal visualisation to facilitate the accurate, human driven analysis of gameplay data. While a great deal of research explores these systems in terms of design, there is not yet a concrete understanding of how users, especially players, interact with these systems in order to extract insight and meaning. Further, it is difficult to adapt existing interaction taxonomies from the infovis literature due to the inherently different nature of game data. As a result, there is no clear understanding of the best way to design systems, especially interactive systems, such that they can support the processes engaged by users when trying to extract insight from data. In this paper, we present the beginnings of a taxonomy for interaction with spatio-temporal game data visualization as the first step towards generating a formal taxonomy and a concrete understanding of the topic.

We conducted a qualitative think-aloud study with seven DotA 2 players, whose experience ranged from 3 to 8 years, in which they performed a series of data analysis and interpretation tasks while interacting with Stratmapper, a spatio-temporal game data visualization system. The result of this study was a preliminary taxonomy for user interaction with spatio-temporal game data visualization that encompasses both observable actions and inferred cognitive processes. We identified seven activities organized into three categories: Data Interaction (consisting of "study positioning to construct context", "study movement to infer decisions", and "seek details to support sense-making"), Sense Making (consisting of "leverage domain knowledge to fill gaps", "form a hypothesis of context and behavior that evolves over time", "pinpoint events to frame understanding"), and Validation (consisting of "review events to confirm hypothesis"). We also discussed a preliminary process model for how users employ these activities as they engage and move between these activities in order to generate a mental model of gameplay. We presented these results along with a discussion of how they relate to existing literature and implications for future research and system design. While there are many avenues for future work, the work presented here is the first step towards a formal, validated, concrete, and generalizable interaction taxonomy for spatio-temporal game data visualization.

\section{ACKNOWLEDGMENTS}

This work is partially supported by the National Science Foundation under Grant Number 1917855 and he authors would like to thank all current members of the project. This material is also based upon work supported by the Office of Naval Research under Contract No. N68335-20-C-0401. Any opinions, findings and conclusions or recommendations expressed in this material are those of the author(s) and do not necessarily reflect the views of the Office of Naval Research.

\section{REFERENCES}

[1] Ana Paula Afonso, Maria Beatriz Carmo, Tiago Gonçalves, and Pedro Vieira. 2019. VisuaLeague: Player performance analysis using spatial-temporal data. Multimedia Tools and Applications 78, 23 (2019), 33069-33090.

[2] Sabbir Ahmad, Andy Bryant, Erica Kleinman, Zhaoqing Teng, Truong-Huy D Nguyen, and Magy Seif El-Nasr. 2019. Modeling Individual and Team Behavior through Spatio-temporal Analysis. In Proceedings of the Annual Symposium on Computer-Human Interaction in Play. 601-612.

[3] Blizzard Entertainment. 1999. StarCraft. [Digital Game].

[4] Brian Bowman, Niklas Elmqvist, and TJ Jankun-Kelly. 2012. Toward visualization for games: Theory, design space, and patterns. IEEE transactions on visualization and computer graphics 18, 11 (2012), 1956-1968.

[5] John L Campbell, Charles Quincy, Jordan Osserman, and Ove K Pedersen. 2013. Coding in-depth semistructured interviews: Problems of unitization and intercoder reliability and agreement. Sociological Methods \& Research 42,3 
(2013), 294-320.

[6] Olivier Cavadenti, Victor Codocedo, Jean-François Boulicaut, and Mehdi Kaytoue. 2016. What did i do wrong in my MOBA game? Mining patterns discriminating deviant behaviours. In Data Science and Advanced Analytics (DSAA), 2016 IEEE International Conference on. IEEE, 662-671.

[7] Sven Charleer, Kathrin Gerling, Francisco Gutiérrez, Hans Cauwenbergh, Bram Luycx, and Katrien Verbert. 2018. Real-time dashboards to support esports spectating. In Proceedings of the 2018 Annual Symposium on Computer-Human Interaction in Play. 59-71.

[8] Jacob Cohen. 1960. A coefficient of agreement for nominal scales. Educational and psychological measurement 20, 1 (1960), 37-46.

[9] Anders Drachen, Rafet Sifa, Christian Bauckhage, and Christian Thurau. 2012. Guns, swords and data: Clustering of player behavior in computer games in the wild. In 2012 IEEE conference on Computational Intelligence and Games (CIG) IEEE, 163-170.

[10] Joshua A Eaton, David J Mendonça, and Matthew-Donald D Sangster. 2018. Attack, Damage and Carry: Role Familiarity and Team Performance in League of Legends. In Proceedings of the Human Factors and Ergonomics Society Annual Meeting, Vol. 62. SAGE Publications Sage CA: Los Angeles, CA, 130-134.

[11] Joshua A Eaton, Matthew-Donald D Sangster, Molly Renaud, David J Mendonca, and Wayne D Gray. 2017. Carrying the Team: The Importance of One Player's Survival for Team Success in League of Legends. In Proceedings of the Human Factors and Ergonomics Society Annual Meeting, Vol. 61. SAGE Publications Sage CA: Los Angeles, CA, 272-276.

[12] Christoph Eggert, Marc Herrlich, Jan Smeddinck, and Rainer Malaka. 2015. Classification of player roles in the team-based multi-player game dota 2. In International Conference on Entertainment Computing. Springer, 112-125.

[13] Magy Seif El-Nasr, Anders Drachen, and Alessandro Canossa. 2013. Game analytics. Springer.

[14] Alex Endert, M Shahriar Hossain, Naren Ramakrishnan, Chris North, Patrick Fiaux, and Christopher Andrews. 2014. The human is the loop: new directions for visual analytics. Fournal of intelligent information systems 43, 3 (2014), 411-435.

[15] Ana Figueiras. 2015. Towards the understanding of interaction in information visualization. In 2015 19th International Conference on Information Visualisation. IEEE, 140-147.

[16] André R Gagné, Magy Seif El-Nasr, and Chris D Shaw. 2011. A Deeper Look at the use of Telemetry for Analysis of Player Behavior in RTS Games. In International Conference on Entertainment Computing. Springer, 247-257.

[17] Helen Gavin. 2008. Thematic analysis. Understanding research methods and statistics in psychology (2008), 273-282.

[18] Tiago Gonçalves, Pedro Vieira, Ana Paula Afonso, Maria Beatriz Carmo, and Tiago Moucho. 2018. Analysing player performance with animated maps. In 2018 22nd international conference information visualisation (IV). IEEE, 103-109.

[19] Nour Halabi, Günter Wallner, and Pejman Mirza-Babaei. 2019. Assessing the impact of visual design on the interpretation of aggregated playtesting data visualization. In Proceedings of the Annual Symposium on Computer-Human Interaction in Play. 639-650.

[20] William A Hamilton, Oliver Garretson, and Andruid Kerne. 2014. Streaming on twitch: fostering participatory communities of play within live mixed media. In Proceedings of the SIGCHI conference on human factors in computing systems. 1315-1324.

[21] Dandan Huang, Melanie Tory, Bon Adriel Aseniero, Lyn Bartram, Scott Bateman, Sheelagh Carpendale, Anthony Tang, and Robert Woodbury. 2014. Personal visualization and personal visual analytics. IEEE Transactions on Visualization and Computer Graphics 21, 3 (2014), 420-433.

[22] Nithesh Javvaji, Casper Harteveld, and Magy Seif El-Nasr. 2020. Understanding Player Patterns by Combining Knowledge-Based Data Abstraction with Interactive Visualization. In Proceedings of the Annual Symposium on ComputerHuman Interaction in Play.

[23] Yea-Seul Kim, Katharina Reinecke, and Jessica Hullman. 2017. Explaining the gap: Visualizing one's predictions improves recall and comprehension of data. In Proceedings of the 2017 CHI Conference on Human Factors in Computing Systems. 1375-1386.

[24] Erica Kleinman, Sabbir Ahmad, Zhaoqing Teng, Andy Bryant, Truong-Huy D. Nguyen, Casper Harteveld, and Magy Seif El-Nasr. 2020. "And Then They Died": Using Action Sequences for Data Driven, Context Aware Gameplay Analysis. In International Conference on the Foundations of Digital Games (Bugibba, Malta) (FDG '20). Association for Computing Machinery, New York, NY, USA, Article 63, 12 pages.

[25] Erica Kleinman and Magy Seif El-Nasr. 2020. Interactive Visualization For Strategy Acquisition In Esports Spectatorship. In Seegames Workshop, CHI 2020.

[26] Athanasios Vasileios Kokkinakis, Simon Demediuk, Isabelle Nölle, Oluseyi Olarewaju, Sagarika Patra, Justus Robertson, Peter York, Alan Pedrassoli Pedrassoli Chitayat, Alistair Coates, Daniel Slawson, et al. 2020. DAX: Data-Driven Audience Experiences in Esports. In ACM International Conference on Interactive Media Experiences. 94-105.

[27] Simone Kriglstein, Günter Wallner, and Margit Pohl. 2014. A user study of different gameplay visualizations. In Proceedings of the SIGCHI Conference on Human Factors in Computing Systems. 361-370. 
[28] Yen-Ting Kuan, Yu-Shuen Wang, and Jung-Hong Chuang. 2017. Visualizing real-time strategy games: The example of starcraft ii. In 2017 IEEE Conference on Visual Analytics Science and Technology (VAST). IEEE, 71-80.

[29] J Richard Landis and Gary G Koch. 1977. The measurement of observer agreement for categorical data. biometrics (1977), 159-174.

[30] Sukwon Lee, Sung-Hee Kim, Ya-Hsin Hung, Heidi Lam, Youn-ah Kang, and Ji Soo Yi. 2015. How do people make sense of unfamiliar visualizations?: A grounded model of novice's information visualization sensemaking. IEEE transactions on visualization and computer graphics 22,1 (2015), 499-508.

[31] Clayton Lewis. 1982. Using the" thinking-aloud" method in cognitive interface design. IBM TJ Watson Research Center Yorktown Heights, NY.

[32] Zhicheng Liu and John Stasko. 2010. Mental models, visual reasoning and interaction in information visualization: A top-down perspective. IEEE transactions on visualization and computer graphics 16, 6 (2010), 999-1008.

[33] Eva Mayr, Günther Schreder, Michael Smuc, and Florian Windhager. 2016. Looking at the representations in our mind: Measuring mental models of information visualizations. In Proceedings of the Sixth Workshop on Beyond Time and Errors on Novel Evaluation Methods for Visualization. 96-103.

[34] Ben Medler. 2011. Player dossiers: Analyzing gameplay data as a reward. Game Studies 11, 1 (2011).

[35] Ben Medler and Brian Magerko. 2011. Analytics of play: Using information visualization and gameplay practices for visualizing video game data. Parsons fournal for Information Mapping 3, 1 (2011), 1-12.

[36] Dinara Moura, Magy Seif el Nasr, and Christopher D Shaw. 2011. Visualizing and understanding players' behavior in video games: discovering patterns and supporting aggregation and comparison. In Proceedings of the 2011 ACM SIGGRAPH symposium on video games. ACM, 11-15.

[37] Truong-Huy D Nguyen, Magy Seif El-Nasr, and Alessandro Canossa. 2015. Glyph: Visualization Tool for Understanding Problem Solving Strategies in Puzzle Games.. In FDG.

[38] The Language Archive Nijmegen: Max Planck Institute for Psycholinguistics. 2020. ELAN (Version 6.0) [Computer software]. Retrieved from https://archive.mpi.nl/tla/elan.

[39] Hao Yi Ong, Sunil Deolalikar, and Mark Peng. 2015. Player Behavior and Optimal Team Composition for Online Multiplayer Games. arXiv preprint arXiv:1503.02230 (2015).

[40] Ernesto Panadero. 2017. A review of self-regulated learning: Six models and four directions for research. Frontiers in psychology 8 (2017), 422.

[41] Robert E Patterson, Leslie M Blaha, Georges G Grinstein, Kristen K Liggett, David E Kaveney, Kathleen C Sheldon, Paul R Havig, and Jason A Moore. 2014. A human cognition framework for information visualization. Computers \& Graphics 42 (2014), 42-58.

[42] Sean Penney, Jonathan Dodge, Claudia Hilderbrand, Andrew Anderson, Logan Simpson, and Margaret Burnett. 2018 Toward foraging for understanding of StarCraft agents: An empirical study. In 23rd International Conference on Intelligent User Interfaces. 225-237.

[43] Peter Pirolli and Stuart Card. 2005. The sensemaking process and leverage points for analyst technology as identified through cognitive task analysis. In Proceedings of international conference on intelligence analysis, Vol. 5. McLean, VA, USA, 2-4.

[44] Nataliia Pobiedina, Julia Neidhardt, Maria del Carmen Calatrava Moreno, Laszlo Grad-Gyenge, and Hannes Werthner 2013. On successful team formation: Statistical analysis of a multiplayer online game. In 2013 IEEE 15th Conference on Business Informatics. IEEE, 55-62.

[45] Zachary Pousman, John Stasko, and Michael Mateas. 2007. Casual information visualization: Depictions of data in everyday life. IEEE transactions on visualization and computer graphics 13, 6 (2007), 1145-1152.

[46] Daniel Ramirez-Cano, Simon Colton, and Robin Baumgarten. 2010. Player classification using a meta-clustering approach. In Proceedings of the 3rd Annual International Conference Computer Games, Multimedia \& Allied Technology. 297-304.

[47] Lei Ren, Jin Cui, Yi Du, and Guozhong Dai. 2013. Multilevel interaction model for hierarchical tasks in information visualization. In Proceedings of the 6th International Symposium on Visual Information Communication and Interaction. $11-16$.

[48] Riot Games. 2009. League of Legends. [Digital Game].

[49] Sara Rodrigues. 2020. Unexplored and familiar: experiencing interactive spatio-temporal visualization. In 2020 15th Iberian Conference on Information Systems and Technologies (CISTI). IEEE, 1-6.

[50] Sara Rodrigues and Ana Figueiras. [n.d.]. There and then: interacting with spatio-temporal visualization. ([n.d.]).

[51] Laura Rook. 2013. Mental models: A robust definition. The learning organization (2013).

[52] Alain Saas, Anna Guitart, and Africa Periánez. 2016. Discovering playing patterns: Time series clustering of free-to-play game data. In 2016 IEEE Conference on Computational Intelligence and Games (CIG). IEEE, 1-8.

[53] Dominik Sacha, Andreas Stoffel, Florian Stoffel, Bum Chul Kwon, Geoffrey Ellis, and Daniel A Keim. 2014. Knowledge generation model for visual analytics. IEEE transactions on visualization and computer graphics 20, 12 (2014), 1604-1613. 
[54] Johnny Saldaña. 2021. The coding manual for qualitative researchers. SAGE Publications Limited.

[55] Kari Sandouka. 2019. Interactive Visualizations: A Literature Review. MWAIS 2019 Proceedings (2019), 8.

[56] Kamran Sedig, Paul Parsons, and Alex Babanski. 2012. Towards a characterization of interactivity in visual analytics. f. Multim. Process. Technol. 3, 1 (2012), 12-28.

[57] Magy Seif El-Nasr and Erica Kleinman. 2020. Data-Driven Game Development: Ethical Considerations. In International Conference on the Foundations of Digital Games. 1-10.

[58] Ben Shneiderman. 1996. The eyes have it: A task by data type taxonomy for information visualizations. In Proceedings 1996 IEEE symposium on visual languages. IEEE, 336-343.

[59] Max Sjöblom and Juho Hamari. 2017. Why do people watch others play video games? An empirical study on the motivations of Twitch users. Computers in human behavior 75 (2017), 985-996.

[60] Adam Stern. 2020. Top 10 Esports Games of 2020 by Total Winnings. Retrieved from https://esportsobserver.com/top10games-2020-total-winnings/.

[61] Eliane RA Valiati, Marcelo S Pimenta, and Carla MDS Freitas. 2006. A taxonomy of tasks for guiding the evaluation of multidimensional visualizations. In Proceedings of the 2006 AVI workshop on Beyond time and errors: novel evaluation methods for information visualization. 1-6.

[62] Wesley van den Broek, Günter Wallner, and Regina Bernhaupt. 2019. Modata-Improving Dota 2 Experience and Spectatorship through Tangible Gameplay Visualization. In Extended Abstracts of the Annual Symposium on ComputerHuman Interaction in Play Companion Extended Abstracts. 723-730.

[63] Günter Wallner, Nour Halabi, and Pejman Mirza-Babaei. 2019. Aggregated visualization of playtesting data. In Proceedings of the 2019 CHI Conference on Human Factors in Computing Systems. 1-12.

[64] Günter Wallner and Simone Kriglstein. 2012. A spatiotemporal visualization approach for the analysis of gameplay data. In Proceedings of the SIGCHI conference on human factors in computing systems. 1115-1124.

[65] Günter Wallner and Simone Kriglstein. 2013. Visualization-based analysis of gameplay data-a review of literature. Entertainment Computing 4, 3 (2013), 143-155.

[66] Guenter Wallner and Simone Kriglstein. 2016. Visualizations for retrospective analysis of battles in team-based combat games: A user study. In Proceedings of the 2016 Annual Symposium on Computer-Human Interaction in Play. 22-32.

[67] Günter Wallner and Simone Kriglstein. 2020. Multivariate Visualization of Game Metrics: An Evaluation of Hexbin Maps. In Proceedings of the Annual Symposium on Computer-Human Interaction in Play. 572-584.

[68] Günter Wallner, Simone Kriglstein, Florian Gnadlinger, Michael Heiml, and Jochen Kranzer. 2014. Game user telemetry in practice: A case study. In Proceedings of the 11th Conference on Advances in Computer Entertainment Technology. 1-4.

[69] GÜNTER WALLNER, MARNIX VAN WIJLAND, REGINA BERNHAUPT, and SIMONE KRIGLSTEIN. 2021. What Players Want: Information Needs of Players on Post-Game Visualizations. (2021).

[70] M Adil Yalçin, Niklas Elmqvist, and Benjamin B Bederson. 2016. Cognitive stages in visual data exploration. In Proceedings of the Sixth Workshop on Beyond Time and Errors on Novel Evaluation Methods for Visualization. 86-95.

[71] Ji Soo Yi, Youn ah Kang, John Stasko, and Julie A Jacko. 2007. Toward a deeper understanding of the role of interaction in information visualization. IEEE transactions on visualization and computer graphics 13, 6 (2007), 1224-1231.

[72] Ji Soo Yi, Youn-ah Kang, John T Stasko, and Julie A Jacko. 2008. Understanding and characterizing insights: how do people gain insights using information visualization?. In Proceedings of the 2008 Workshop on BEyond time and errors: novel evaLuation methods for Information Visualization. 1-6.

[73] Caroline Ziemkiewicz, Steven Gomez, and David Laidlaw. 2012. Analysis within and between graphs: Observed user strategies in immunobiology visualization. In Proceedings of the SIGCHI Conference on Human Factors in Computing Systems. 1655-1658.

Received February 2021 ; revised June 2021 ; accepted July 2021 